\title{
Differential diagnosis and management of focal pulmonary ground-glass opacities
}

\section{To the Editors:}

In a recent article printed in the European Respiratory Journal, INFANTE et al. [1] validated a stepwise approach including oral antibiotics, follow-up computed tomography and computed tomography-guided biopsy for the differential diagnosis and management of focal pulmonary ground-glass opacities (GGOs). Although a retrospective study, their work provides critical information on discrimination and management of focal GGOs. However, some of the results in the article did not fully support their conclusions.

One of the major conclusions in the study by INFANTE et al. [1] was that the stepwise approach increased the diagnostic specificity and reduced time to definitive diagnosis. They recommended response to antibiotic trial as the first management step. After a course of oral antibiotics, five (12.5\%) patients with inflammatory lesions were ruled out. However, six patients in their study were evaluated with lesions retrospectively visible in earlier computed tomography scans and oral antibiotics were not given. Since the patients with regression were outnumbered by those without treatment, the possibility that all five patients with regression did not receive antibiotic treatment could not be excluded. If all the patients with regression lesions did receive oral antibiotics treatment, the response rate could be elevated to $14.7 \%$ (five out of 34 patients). Because this is a central part of their conclusion, clarification of the question may be helpful. INFANTE et al. [1] assumed that lesions with regression after antibiotics treatment were benign GGOs. However, a decrease in size with the appearance of solid components during follow-up has been reported in bronchioloalveolar carcinoma (BAC) and adenocarcinoma with mixed subtype [2]. Therefore, analysis of the regression patterns and further follow-up of patients with suspicious appearance are mandatory. The correlation between antibiotic treatment, patterns of regression and histology of GGOs needs a larger study for validation. BAC is known to have a relatively slow growth pattern and indolent clinical course. Some BAC may even be stable in size and appearance for years. The 10 patients with undetermined lesions may also have a risk of malignancy.

Radiographical presentations of BAC have been reported in the literature. INFANTE et al. [1] presented that nonpolygonal shape, apparent radial growth and clear-cut margins were associated with malignant histology. The association between a solid component and lung cancer was nonsignificant in their study. However, correlation of a solid component with increased suspicion for invasive adenocarcinoma has been reported [2,3]. An increase in size of a GGO, development of a solid component within a GGO and an increase in the solid component of a mixed-attenuation lesion on serial computed tomography are features suggesting malignancy $[2,3]$. Since follow-up computed tomography was performed 40-60 days after antibiotic treatment, comparison of the radiographical presentations may provide a more powerful feature to improve the prediction of malignancy in their study.

The other conclusion of their study is that segmentectomy might be the ideal resection volume for focal GGOs. Although BAC has a low invasive potential and excellent prognosis after surgical excision, the indications of sublobar resections including wedge resection and segmentectomy remain controversial [4-8]. Aerogenous spread, a BAC hallmark, is related to the predominantly local recurrences after sublobar resection. The lack of large, randomised series with long-term follow-up makes the precise role of sublobar resections unclear. INFANTE et al. [1] advocated segmentectomy for focal GGOs, mainly based on a retrospective and nonrandomised study reporting an equivalent cure rate of small early-stage tumours with anatomic segmentectomy and standard lobectomy [6]. However, six of the seven patients with BAC in their study underwent lobectomy, instead of segmentectomy. Only four out of all 40 patients received segmentectomy. Furthermore, two patients in their study developed recurrent lung cancer in the ipsilateral lung. One of them had recurrence 6 months following right lower lobectomy for stage I BAC. We wonder, what the cause is leading to the gap between their advocate and true practice. Both their practice experience and results did not provide sufficient support for their conclusion to recommend segmentectomy. Until the equivalent cure rate with sublobar resections and standard lobectomy is confirmed by ongoing randomised clinical trials, lobectomy and mediastinal lymph node sampling or dissection remain the treatment of choice for cure, including T1 BAC [4, 5].

The data presented by INFANTE et al. [1] in figure 1 and the text are not consistent. For example, the number of patients with no regression, with lung cancer and the number of patients with resection are inconsistent. Figure 1 is also difficult to interpret because the number of patients is confusing and its structure is not well arranged. Publication of a paper with such defects is discouraging.

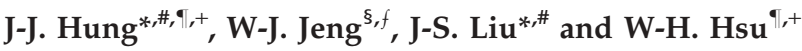 *Cathay General Hospital, "Fu Jen Catholic University, "Taipei Veterans General Hospital, 'National Yang-Ming University, Taipei, ${ }^{\S}$ Dept of Internal Medicine, Chang Gung Memorial Hospital, and ${ }^{f}$ School of Medicine, Chang Gung University, Taoyuan, Taiwan.}

Correspondence: J-J. Hung, Division of Thoracic Surgery, Dept of Surgery, Cathay General Hospital, No. 280, Sec. 4, Renai Road, Taipei 106, Taiwan. E-mail: bradley.hung@gmail.com

\section{Statement of Interest: None declared.}

\section{REFERENCES}

1 Infante M, Lutman RF, Imparato S, et al. Differential diagnosis and management of focal ground-glass opacities. Eur Respir J 2009; 33: 821-827. 
2 Kakinuma R, Ohmatsu H, Kaneko M, et al. Progression of focal pure ground-glass opacity detected by low-dose helical computed tomography screening for lung cancer. J Comput Assist Tomogr 2004; 28: 17-23.

3 Takashima S, Maruyama Y, Hasegawa M, et al. CT findings and progression of small peripheral lung neoplasms having a replacement growth pattern. AJR Am J Roentgenol 2003; 180: 817-826.

4 Ginsberg RJ, Rubenstein LV. Randomized trial of lobectomy versus limited resection for T1N0 non-small cell lung cancer. Ann Thorac Surg 1995; 60: 615-622.

5 Rusch VW, Tsuchiya R, Tsuboi M, et al. Surgery for bronchioloalveolar carcinoma and "very early" adenocarcinoma: an evolving standard of care? J Thorac Oncol 2006; 1: Suppl. 9, S27-S31.

6 Okada M, Koike T, Higashiyama M, et al. Radical sublobar resection for small-sized non-small cell lung cancer: a multicenter study. J Thorac Cardiovasc Surg 2006; 132: 769-775.

7 Yoshida J, Nagai K, Yokose T, et al. Limited resection trial for pulmonary ground-glass opacity nodules: fifty-case experience. J Thorac Cardiovasc Surg 2005; 129: 991-996.

8 Nakata M, Sawada S, Yamashita M, et al. Objective radiologic analysis of ground-glass opacity aimed at curative limited resection for small peripheral non-small cell lung cancer. J Thorac Cardiovasc Surg 2005; 129: 1226-1231.

DOI: $10.1183 / 09031936.00085709$

From the authors:

In our paper [1], we summarised the expertise and practical guidelines we have built in recent years for the differential diagnosis and management of focal ground-glass opacities (GGOs) detected by spiral computed tomography (CT), either in a screening programme or incidentally.

As J-J. Hung and co-workers rightly point out, malignant GGOs may show a mixed evolution pattern over time with a decrease in their overall size but an actual increase of the central solid component. This should not be mistaken for a regression, but rather for progression to solid adenocarcinoma [2]. Regression in response to antibiotic trial was defined by us as fading or disappearance of the entire lesion, which occurred in five $(12.5 \%)$ cases.

Six patients were evaluated retrospectively, as their GGOs were visible on previous CT scans months before the lesion was actually reported. Antibiotics were not prescribed at that point as we did not expect them to have an effect on such lesions. Although this was an assumption, we considered five out of 40 or $12.5 \%$ an appropriate effect size measure to avoid overestimating the observed benefit.

Lesions that are not submitted to biopsy and do not disappear nor apparently progress may eventually turn out to be very slow-growing cancers over time, and we believe that follow-up should be continued at yearly intervals beyond the limit of three years that we have arbitrarily chosen to declare a lesion to be benign. Further observation of our patients may provide interesting information in the future.

The association between a solid component and lung cancer did not reach statistical significance in this series due to lack of adequate power, but this per se does not contradict the observation that a solid component within a GGO suggests malignancy. The positive predictive value for lung cancer of a solid density within the GGO was in fact $76 \%$ in our patients; in other words, if a solid component was apparent on CT, the lesions turned out to be malignant three out of four times.

In this series, as of March 2008 only 19 out of 40 patients had undergone surgical resection. Three patients with bronchioloalveolar carcinoma (BAC) had a segmentectomy and two more had a combination of lobectomy plus sublobar resections for multiple mixed-type adenocarcinomas in different lobes; therefore, five out of 18 patients had one or more segmental or sublobar resections for malignant GGOs. In addition, one patient had a segmentectomy only to confirm that the lesion was indeed atypical adenomatous hyperplasia (as it showed a small central solid component). Therefore, six (32\%) out of 19 patients had sublobar resections alone or in combination, a proportion that has since been increasing.

We suggest that segmentectomy may be appropriate for focal GGOs mainly based on the abundant literature favouring sublobar resections in these patients [3, 4], and because we believe it is a reasonable compromise operation that allows for adequate surgical margins, hilar nodal dissection and lung function preservation. We acknowledge that firm evidence (derived from large randomised trials) in favour of this approach is lacking, but it should also be remembered that evidence in favour of a priori lobectomy for T1N0 lung cancer is still largely derived from a single randomised trial carried out in 1995 by the Lung Cancer Study Group on patients harbouring solid nodules of various nonsmall cell types [5]. Compared with solid lung cancers, BAC and mixed-type

Asymptomatic patients with localised GGOs $(n=40)$

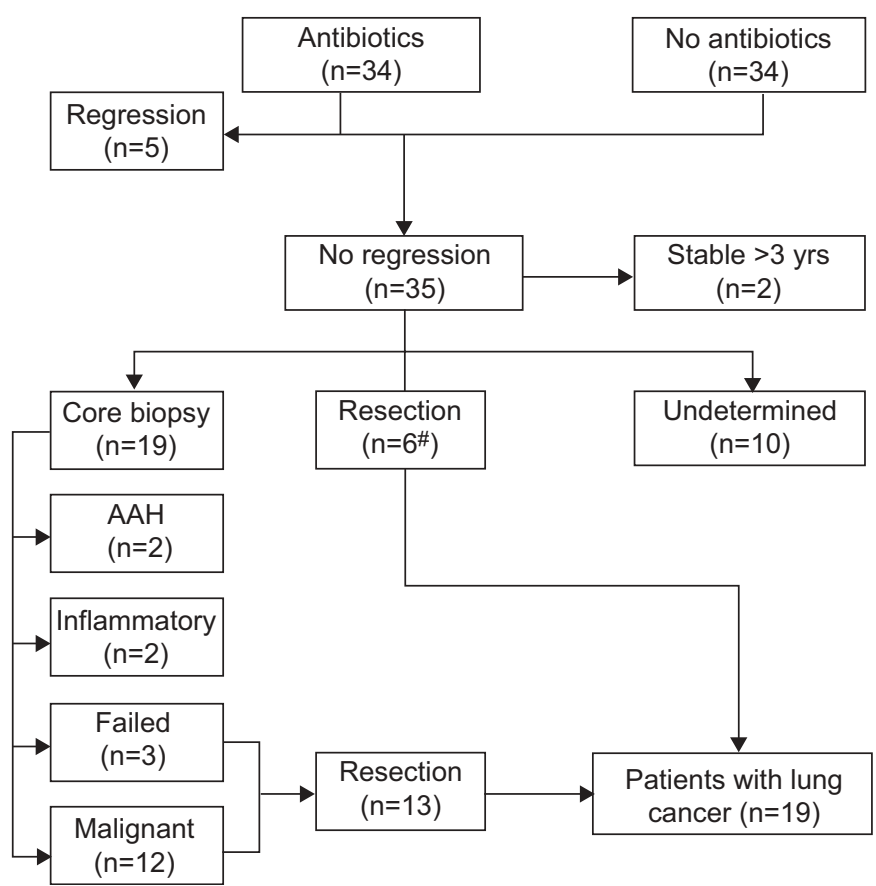

FIGURE 1. Diagram of patient management procedures. In six patients, the lesion was retrospectively visible in an earlier computed tomography scan, and antibiotics were not administered. GGO: ground-glass opacity; $\mathrm{AAH}$ : atypical adenomatous hyperplasia. \#: one patient was operated on twice for a double malignant GGO 